Francophonies d'Amérique

\title{
Une nouvelle ressource collective pour des communautés francophones minoritaires durables : le centre scolaire-communautaire en Acadie du Nouveau-Brunswick et au Canada
}

\section{Greg Allain}

Numéro 22, automne 2006

Urbanité et durabilité des communautés francophones du Canada

URI : https://id.erudit.org/iderudit/1005376ar

DOI : https://doi.org/10.7202/1005376ar

Aller au sommaire du numéro

Éditeur(s)

Les Presses de l'Université d'Ottawa

Centre de recherche en civilisation canadienne-française

\section{ISSN}

1183-2487 (imprimé)

1710-1158 (numérique)

Découvrir la revue

Citer cet article

Allain, G. (2006). Une nouvelle ressource collective pour des communautés francophones minoritaires durables : le centre scolaire-communautaire en Acadie du Nouveau-Brunswick et au Canada. Francophonies d'Amérique, (22), 13-35. https://doi.org/10.7202/1005376ar

Tous droits réservés (C) Les Presses de l'Université d'Ottawa et Centre de recherche en civilisation canadienne-française, 2006
Ce document est protégé par la loi sur le droit d'auteur. L'utilisation des services d'Érudit (y compris la reproduction) est assujettie à sa politique d'utilisation que vous pouvez consulter en ligne.

https://apropos.erudit.org/fr/usagers/politique-dutilisation/ 


\title{
UNE NOUVELLE RESSOURCE COLLECTIVE POUR DES COMMUNAUTÉS FRANCOPHONES MINORITAIRES DURABLES : LE CENTRE SCOLAIRE-COMMUNAUTAIRE EN ACADIE DU NOUVEAU-BRUNSWICK ET AU CANADA
}

\author{
Greg Allain \\ Université de Moncton
}

$\mathrm{C}_{\mathrm{n}}$ mai 2005 , le $73^{\mathrm{e}}$ Congrès de l'Association francophone pour le savoir (ACFAS)

- se tenait à l'Université du Québec à Chicoutimi autour du thème "Innovations durables ». Pour sa part, le Réseau de la recherche sur la francophonie canadienne y tenait son colloque annuel, la thématique retenue étant "Durabilité de nos communautés : de nouvelles ressources et de nouveaux défis $"$.

La pertinence de cette thématique devrait être évidente : même s'il y a un débat sur la vitalité des communautés francophones minoritaires au Canada - nous y reviendrons - la question de la durabilité de cette vitalité communautaire est une question légitime, et partant, celle des ressources pour assurer sa viabilité à long terme, ou, pourrait-on dire, pour rendre possible le développement durable de ces communautés.

Mais avant d'aborder un des outils collectifs identifiés comme une ressource significative en ce sens, puisque nous avons évoqué la notion de développement durable dans le contexte qui nous occupe, commençons par décortiquer brièvement cette notion qui fait l'objet de vifs débats depuis son apparition il y a une vingtaine d'années.

\section{Le développement durable : un concept populaire et contesté, à géométrie variable}

Les idées qui sous-tendent le terme " développement durable » auraient commencé à circuler au cours des années 70 et 80 (Vaillancourt, 2004:38-39)2, mais ce qui propulsera littéralement cette notion sur la scène mondiale sera la publication du très médiatisé rapport de la Commission des Nations Unies sur l'environnement et le développement, Our Common Future, en 1987, et de sa traduction française Notre avenir à tous, l'année suivante, mieux connu comme le Rapport Brundtland, du nom de la présidente de la commission, mise sur pied en 1983 par l'Assemblée générale de l'ONU pour compenser l'échec total de la Conférence de Nairobi sur l'environnement en 1982 (voir l'analyse de Vaillancourt, 2004 : 37-38). Le concept de développement durable est au cœur de ce rapport célèbre, et il inspirera grandement entre autres les travaux du Sommet de la terre de Rio en 1992 et se verra concrétisé à l'échelle mondiale avec les accords de la Conférence de Rio en 2002. 
Le Rapport Brundtland définit ainsi le développement durable: "Un développement qui répond aux besoins du présent sans compromettre la capacité des générations futures de répondre aux leurs " (Report of the..., 1987 : 51). Il s'agirait de " la définition [...] la plus couramment acceptée du terme " (Guay, 2004:4). Mais il y a un paradoxe autour de cette notion : autant elle a fait le tour du monde et est acceptée par de larges pans de l'opinion publique (y compris de la plupart des gouvernements, des organismes internationaux, comme la Banque mondiale et le Fonds monétaire international (FMI), ainsi que des dirigeants de grands conglomérats), autant elle fait l'objet de critiques à la fois répandues et pointues. On lui reproche sa foncière ambiguïté ${ }^{3}$ (par exemple, la notion de besoins reste très vague, et les moyens proposés semblent timides et dépassés par rapport à l'ampleur de la crise environnementale mondiale diagnostiquée), qui en fait un " concept contestable " (Zaccaï, 2002 : 35), au même titre que d'autres concepts politiques qui affirment de grands principes généraux (comme le progrès, la modernisation, la démocratie...) sans préciser leurs conditions de réalisation, y compris des réorientations radicales si nécessaire.

La notion de développement durable est donc floue (Daly, $1990: 1$ ), ce qui lui permet de devenir une auberge espagnole sur le plan conceptuel. Comme le souligne l'économiste français Serge Latouche, l'unanimité même dont elle fait l'objet la rend suspecte, car " une clef qui ouvre toutes les portes est une mauvaise clef " (2004: 66$67)^{4}$. Il faut aussi prendre conscience, c'est en tout cas ce que plusieurs experts lui reprochent, qu'elle constitue un compromis, voire un recul par rapport à des positions antérieures plus radicales, comme la halte à la croissance proposée par le Club de Rome (Meadows et al., 1972) et la revendication d'un véritable écodéveloppement (Sachs, 1980).

Ainsi donc, au-delà d'une fréquente confusion terminologique (d'aucuns par exemple parlent de développement soutenable, viable ou durable et équitable) ${ }^{5}$, l'idée de développement durable constituerait au jugement de certains un réel oxymore, c'est-àdire une contradiction dans les termes (Latouche, $2004: 51$ ) représentant un autre cas de développement "à particule " (comme le développement social, le développement humain, le développement local...) (Latouche, 2004 : 31-72). Une grande partie du problème vient du fait que, malgré les discours officiels et la rhétorique, on associe encore la notion de développement à celle de croissance économique. Dit autrement, même si l'on affirme que le développement (qualitatif) est beaucoup plus que la simple croissance (quantitative), on continue de croire que cette dernière est indispensable à l'atteinte du développement. C'est sans doute ce qui explique que le concept de développement durable rallie autant de suffrages, tant du côté des industriels que de celui des plus grandes instances capitalistes internationales comme la Banque mondiale et le FMI, au point de devenir le discours dominant en la matière (Gendron, 2004 : 65-72).

En fait, on se trouve peut-être dorénavant placé devant deux paradigmes opposés, que le sociologue Ronald Babin nomme "la modernisation écologique de l'économie " qui propose une vision réformiste du statu quo, et «la modernisation sociétale des 
conditions d'existence " qui tranche avec le système actuel en faveur de changements structurels obéissant à une logique communautaire et de solidarité (Babin, 2004). Une recherche récente menée en 2000 auprès de l'élite économique québécoise du "Top 50 " de la revue Commerce révèle une polarisation semblable : si plusieurs de ces importants gens d'affaires acceptent d'emblée l'idée du développement durable, ils ont évidemment tendance à l'interpréter à leur façon, et ils adhèrent somme toute à une version conservatrice de celle-ci. S'ils reconnaissent l'importance de l'enjeu écologique, ils se placent aux antipodes des écologistes quant à leur diagnostic des causes du phénomène (allant jusqu'à blâmer la surpopulation ainsi que les pays du Sud pour l'épuisement des ressources et même pour la pollution!), de même que des solutions à envisager (la compétition impitoyable entraînée par la mondialisation milite contre des réglementations étatiques trop poussées). L'auteure de l'étude, la sociologue Corinne Gendron, en tire la conclusion suivante :

Ainsi, malgré la reconnaissance de l'enjeu de la protection de l'environnement par les dirigeants, il existe un clivage irréductible entre leur discours et celui des écologistes. C'est ce clivage, profond, irréductible et révélateur d'un rapport social, qui est au cœur des débats entourant la définition du développement durable. À l'instar de la reconnaissance de la problématique environnementale, l'adhésion au projet de développement durable par les dirigeants se fait sur le mode de leurs intérêts et de leur position en tant qu'acteur social. Au fur et à mesure que les dirigeants s'approprient le concept de développement durable, ce dernier est modelé et redéfini en fonction des logiques propres à la construction des représentations sociales (2004:69-70).

Comme les acteurs sociaux dominants ont généralement le pouvoir d'imposer leur vision des choses à l'ensemble de la société, la chercheure fait l'hypothèse qu'un compromis sera éventuellement atteint grâce à une alliance entre la frange éclairée des gens d'affaires et la frange modérée des environnementalistes (Gendron, 2004 : 75-76).

Concluons cette courte incursion sur le terrain du développement durable avec deux constats, l'un d'une philosophe québécoise, l'autre d'un économiste français :

L'apport des considérations environnementales est celui d'un correcteur des dommages du modèle de l'économie libérale. Le concept de développement durable vient ainsi réaffirmer l'universalité du développement des sociétés humaines à partir d'un schéma occidental laissant fort peu de place à des modèles alternatifs ou autres, tant sur le plan économique politique ou culturel. Si l'on peut louer que les préoccupations environnementales soient enfin prises en compte par le modèle de l'économie libérale, il n'en reste pas moins que le concept de développement durable véhicule davantage des valeurs et des bons sentiments qu'un système politique différent qui s'attaque 
aux rapports de force et aux finalités du modèle économique et politique en présence (Parizeau, 2004 : 310-311).

Et plus loin, Marie-Hélène Parizeau ajoute :

Parce qu'il propose avant tout des ajustements au modèle de l'économie libérale occidentale qui domine sans rival la scène économico-politique, le concept de développement durable n'offre guère plus qu'un pis-aller conceptuel sur le plan tant théorique que pratique dans la mesure où il ne remet pas en cause certaines structures du système économique mondial et surtout les rapports de force à l'œuvre. Il manque donc à ce concept de développement durable les analyses politiques qui permettraient de penser à d'autres choix possibles et à d'autres modèles économiques et sociaux, laissant la diversité des cultures s'exprimer par-delà le cadre juridique international normalisateur (2004: 323-324).

Pour l'auteur français Serge Latouche, spécialiste réputé et grand critique du développement appliqué à toutes les sauces, dont l'ingrédient essentiel demeure encore trop souvent la croissance économique tous azimuts, il faut carrément se "désintoxiquer" collectivement de cette drogue idéologique, "décoloniser nos mentalités » et préparer, en solidarité avec les pays du Sud dans une démarche de justice sociale et écologique dirigée contre "l'omnimarchandisation du monde ", l'anti- ou le post- développement. Cela implique selon l'auteur un changement radical :

Pour survivre ou durer, il est donc urgent d'organiser l'après-développement. Quand on est à Rome et que l'on doit se rendre par le train à Turin, si l'on s'est embarqué par erreur dans la direction de Naples, il ne suffit pas de ralentir la locomotive, de freiner ou même de stopper, il faut descendre et prendre un autre train dans la direction opposée. Pour sauver la planète et assurer un futur acceptable à nos enfants, il ne faut pas seulement modérer les tendances actuelles, il faut carrément sortir du développement et de l'économicisme comme il faut sortir de l'agriculture productiviste qui en est partie intégrante pour en finir avec les vaches folles et les aberrations transgéniques (Latouche, $2004: 102-103$ ).

Comme voies de sortie de "l'imposture " du développement durable, l'auteur avance deux pistes : la décroissance conviviale et la revalorisation des initiatives sociales locales comme piliers d'un nouveau système économique décentralisé visant également à refaire les liens sociaux au sein d'une "société vernaculaire " (Latouche, 2004 : 90-113). 


\section{Le développement durable des communautés francophones minoritaires}

Quelles leçons pouvons-nous tirer de la discussion précédente en ce qui a trait à la durabilité des francophonies canadiennes minoritaires? Évidemment, il faut prendre note de la grande polysémie du concept de développement durable, qui entraine un certain nombre de contradictions derrière l'apparente unanimité dont il fait l'objet. Ce qui nous oblige à préciser ce que nous voulons dire au juste quand nous voulons l'employer. Par exemple, si nous l'appliquons aux communautés francophones minoritaires, qu'est-ce précisément ce que nous aimerions voir durer, est-ce que cela inclut le statu quo? De même, il convient de déterminer les moyens devant être mis de l'avant pour réaliser cette durabilité. Dans notre esprit, il ne s'agit pas de pérenniser la situation prévalente à n'importe quel prix, mais de proposer un modèle de " ressource nouvelle" (relativement parlant) susceptible d'alimenter et de faire croître la vitalité communautaire francophone : il s'agit du concept de centre scolaire-communautaire, une innovation institutionnelle (voir Allain, 2005b) mise sur pied au NouveauBrunswick à la fin des années 70 et au cours des années 80 , et qui a ensuite essaimé ailleurs dans les provinces maritimes et dans le reste du Canada.

Par ailleurs, penchons-nous un instant sur les deux pistes proposées par l'économiste Serge Latouche pour sortir de la crise écologique actuelle : si la première (la décroissance conviviale) nous paraît relever de choix sociétaux, la seconde (la revalorisation des initiatives innovatrices locales) nous semble tout à fait compatible avec le modèle discuté ici. Nos recherches sur les communautés acadiennes minoritaires dans trois villes du Nouveau-Brunswick montrent clairement l'efficacité du modèle du centre scolairecommunautaire comme outil de développement et de vitalité dans ces communautés ${ }^{6}$. À partir de nos données surtout ethnographiques, fondées sur des recherches qualitatives sur le terrain, nous estimons être en mesure de poser un diagnostic plus " optimiste " , ou en tout cas moins pessimiste (il importe évidemment de ne pas occulter les problèmes qui existent) qu'un certain nombre d'auteurs pour qui la survie même des populations francophones minoritaires au Canada est sérieusement compromise. Nous pouvons situer dans ce dernier camp les publications, non seulement des démolinguistes et des statisticiens invoquant le poids négatif des "tendances lourdes", comme celles de Charles Castonguay (1993, 1994, 1996, 2002), mais également celles de certains sociologues, comme Roger Bernard (1990, 1996, 1998), et de politologues, comme Réjean Pelletier (2003). Ce dernier déclarait d'ailleurs récemment :

La situation se détériore pour les francophones canadiens et, plus particulièrement, pour les francophones à l'extérieur du Québec. C'est pourquoi on peut parler d'une politique [la politique fédérale des langues officielles] à caractère symbolique qui n’a aucunement enrayé le déclin des francophones au Canada, ni surtout des francophones hors Québec qui sont condamnés à la marginalité $[\ldots]$ [M]algré l'appui fédéral, on assiste à une 
diminution constante et inexorable des francophones à l'extérieur du Québec $(2003: 37,39)^{8}$.

Et l'auteur de conclure sur ces paroles pour le moins démobilisatrices :

Les minorités francophones apparaissent branchées sur le respirateur artificiel fédéral. Si celui-ci coupe l'oxygène, c'est-à-dire l'argent qu'il fournit, ces minorités sont appelées à disparaître petit à petit $(2003: 41)$.

Comble de malheur pour les communautés francophones minoritaires, elles seraient devenues dépendantes des transfusions fédérales qui témoignent d'un acharnement thérapeutique à leur endroit, et le remède n'est même pas efficace pour combattre adéquatement le mal de l'assimilation linguistique... Il faut dire à la décharge de l'auteur qu'il avait, comme on le fait régulièrement depuis le diagnostic de Richard Joy en 1967, accordé des chances de survie à "la frange contiguë au Québec", en Ontario et au Nouveau-Brunswick, "là où ces communautés forment une masse critique qui peut fournir un rempart contre l'assimilation" (Pelletier, 2003:41).

Encore une fois, nous ne partageons pas de tels propos foncièrement démobilisateurs : nous avons pu constater au contraire, dans les trois communautés que nous avons étudiées jusqu'à maintenant, qu'elles ont fait preuve de beaucoup de capacité organisationnelle pour se donner une complétude institutionnelle, pour reprendre les concepts du sociologue canadien Raymond Breton $(1964,1983,1984)$. Le fruit de leurs luttes se traduit par une remarquable vitalité socioculturelle. Et l'un des grands instruments de cette vitalité a été le centre scolaire-communautaire.

\section{Les trois centres scolaires-communautaires du Nouveau-Brunswick}

Les centres scolaires-communautaires représentent une innovation sociale relativement récente, puisque le premier n'ouvrait ses portes qu'en 1978, à Fredericton, la capitale du Nouveau-Brunswick. Après le Centre communautaire Sainte-Anne, deux autres devaient être créés dans la même province, soit le Centre scolaire-communautaire Samuel-de-Champlain, dans la ville industrielle de Saint-Jean, en 1984, et deux ans plus tard, dans la petite ville de Miramichi, ouvrait le Carrefour Beausoleil.

Mais qu'est-ce qu'un centre scolaire-communautaire? Brièvement nous pourrions dire qu'il s'agit d'un complexe abritant une école et un centre communautaire. Mais c'est plus que cela, car nous savons qu' un ensemble constitue plus que la somme de ses parties. En intégrant en un seul endroit les infrastructures scolaires et communautaires, on rend possibles des synergies qui ne se produiraient jamais si l'on avait une école d'un côté et un centre culturel ou communautaire de l'autre. Avec la nouvelle formule, on crée un espace inédit, où la communauté peut trouver des services de base (scolaires, culturels, religieux, organisationnels, même économiques parfois, comme à Fredericton où l'on a ouvert une Caisse populaire dans le centre), et un lieu vital de rassemblement, de 
rencontre, pour répondre à plusieurs besoins fondamentaux et renforcer l'identité et l'appartenance communautaires. Les espaces communs sont multifonctionnels: par exemple, le gymnase, la cafétéria et la bibliothèque servent à des fins scolaires les jours de semaine et se transforment en lieux communautaires les soirs et les fins de semaine. L'amphithéâtre présente des pièces de la troupe scolaire, de la troupe de théâtre amateur de la communauté ou de troupes professionnelles de passage, sans compter les spectacles d'artistes locaux ou de renommée provinciale ou nationale. Et les dimanches, l'amphithéâtre est le siège de célébrations eucharistiques'. Ces installations plurivalentes permettent l'organisation d'un plus large éventail d'activités (Légère, 1989).

Le centre scolaire-communautaire constitue également le cœur du réseau associatif de la collectivité, fournissant des bureaux pour les organismes, des locaux pour leurs réunions et leurs activités ainsi que du personnel de soutien pour les activités d'animation et d'information ${ }^{10}$, ce qui facilite la création d'associations communautaires et la concertation entre les divers groupes (Légère, 1989). En ce sens, le centre scolairecommunautaire constitue un véritable outil de développement pour les collectivités francophones minoritaires (Légère, 1989).

Le tableau 1 indique quelques caractéristiques de base des trois communautés ayant fait l'objet de nos recherches. Les statistiques montrent la diversité de taille des trois villes, Miramichi étant sensiblement plus petite que les deux autres, mais correspondant sans doute à la taille de plusieurs petits centres du reste du Canada. La population urbaine est en léger déclin à Saint-Jean et à Miramichi, mais la population francophone est en croissance à Fredericton et à Saint-Jean. Avouons-le tout de suite : si la formule du centre scolaire-communautaire a manifestement fait ses preuves et remporte un succès indéniable au Nouveau-Brunswick, elle ne représente pas pour autant une recette infaillible, capable à elle seule de parer à de profondes secousses socioéconomiques pouvant ébranler une communauté. Prenons l'exemple de la ville de Miramichi qui, de même que sa population francophone, a été durement frappée par des fermetures importantes ces dernières années : en 1995, c'était le cas de la mine Heath Steele, où plusieurs Acadiens travaillaient, et où 300 emplois ont été perdus, et de la base militaire de Chatham, où tous les employés ont été mutés ailleurs au Canada quand le ministère de la Défense nationale a décidé de mettre la clé dans la porte. Environ 700 francophones (300 familles), surtout d'origine québécoise, quittaient la région à la suite de cette décision : ils constituaient le tiers de la communauté francophone de Miramichi à l'époque (Poulin, 1992 : 84). Toute une amputation.

De plus, en décembre 2004, la compagnie finlandaise UPM-Kymmene-Miramichi, le plus gros employeur local, annonçait la fermeture de son usine de papier Kraft qui employait 400 travailleurs (le moulin à papier, cependant, avec ses 900 emplois, demeure ouvert). Voilà donc une communauté qui a été décimée par des forces extérieures, sur lesquelles elle n'avait aucun contrôle ${ }^{11}$. Le bénévolat en a évidemment souffert, de même que la participation aux activités. La relève n'est pas facile à assurer : c'est sans doute un problème répandu dans beaucoup de communautés, mais il est ici ressenti de façon très aiguë, à la suite des amputations successives dont cette communauté a fait l'objet. Une 
Tableau 1

Caractéristiques de base des trois centres scolaires-communautaires (CSC) du Nouveau-Brunswick

\begin{tabular}{lrrr}
\hline & Fredericton & Saint-Jean & Miramichi \\
\hline Population totale (2001) & 80685 & 123000 & 18500 \\
$\%$ croissance depuis 1996 & $2,2 \%$ & $-2,4 \%$ & $-3,8 \%$ \\
Population francophone & $5890^{\prime}$ & 5775 & $1700^{2}$ \\
$\%$ croissance depuis 1996 & $13 \%$ & $11 \%$ & $-10,5 \%$ \\
$\%$ de la population totale & $7,3 \%{ }^{3}$ & $4,8 \%$ & $9 \%$ \\
Type d'économie & Services & Secondaire, services & Secondaire, services \\
Début des luttes scolaires & 1960 & 1970 & 1979 \\
Ouverture cSC & 1978 & 1984 & 1986 \\
Nombre d'élèves à & 300 & & 197 \\
l'ouverture & 1000 & 320 & 230 \\
Nombre d'élèves 2004-2005 & 1981 & 500 & $(1938)$ \\
Création de la paroisse & & 1981 & 4 \\
Nombre d'associations & 10 & 11 & 19 (26 en 2004) \\
1981 & 33 & 50 & \\
2001 & & & \\
\hline
\end{tabular}

Sources : Allain et Basque, 2001, 2003, 2005; Allain, 2003.

Notes :

1. 8000 , si on inclut les environs jusqu'à Oromocto, soit le territoire desservi par l'école Sainte-Anne (niveau secondaire).

2. 1900 en incluant les environs de la ville de Miramichi.

3. Si on adopte le territoire élargi décrit à la note 1 , alors les 8000 francophones représentent 7,9 p. 100 de la population totale de 101695 .

des conséquences est que plusieurs des leaders actuels font partie du groupe des activistes ayant déclenché le réveil des forces vives à la fin des années 70 . En plus, environ les deux tiers des organismes, qui sont ailleurs présidés par des bénévoles de la communauté, le sont ici par des membres du personnel scolaire ou communautaire. La petite taille de certains organismes les rend également vulnérables lorsque la personne responsable du groupe doit quitter ses fonctions pour cause de maladie ou d'un nouvel engagement professionnel. Enfin, certains dossiers plus complexes mettent beaucoup plus de temps à progresser qu'ailleurs. Par exemple, la mise sur pied d'une association francophone de gens d'affaires et de professionnels, qui a mis deux ou trois ans à se concrétiser à SaintJean, et qui est en voie de se réaliser à Fredericton après le même laps de temps, a fait l'objet de rencontres, de discussions et de démarches à Miramichi pendant une quinzaine 
d'années: finalement, le 15 mars 2005, naissait le Réseau des gens d'affaires francophones de Miramichi (voir Allain et Basque, 2005 : 278-282).

L'autre dossier, dont l'idée date du début des années 80 , soit avant même l'ouverture $\mathrm{du}$ Carrefour Beausoleil à Miramichi, est celui d'une radio communautaire. Il faut savoir qu'une telle radio peut représenter un moyen de communication tout à fait crucial pour une petite minorité francophone qui essaie de (sur)vivre dans un environnement urbain très majoritairement anglophone; elle peut même s'avérer un instrument vital de rassemblement et de développement du groupe francophone. Il faut savoir également que la réalisation d'un tel projet requiert de nombreuses démarches s'échelonnant sur plusieurs années et mobilisant beaucoup de gens dans la communauté : planification, études de marché, études techniques, campagne de financement, formation d'animateurs, expériences pilotes de diffusion, le tout "couronné " par une demande formelle de licence auprès du Conseil de la radiodiffusion et des télécommunications canadiennes (CRTC), qui peut elle-même exiger plusieurs mois avant d'être à l'ordre du jour, sans compter que la réponse du CRTC n'est pas immédiate. Et si le feu vert est accordé, il faut procéder au réaménagement des locaux et à l'installation des équipements, y compris l'antenne de diffusion. Bref, ce n'est pas une mince affaire! La communauté acadienne de Fredericton a commencé des démarches en ce sens en 1990, et c'est sept ans plus tard que Radio CJPN ("Ça joue pour nous") entrait en ondes en août 1997 (Allain et Basque, 2003 : 417-436). À Saint-Jean, c'est vers l'an 2000 que les démarches commencèrent, et l'approbation du CRTC ayant été accordée en 2005, la "Radio Baie de la brise " doit commencer ses émissions régulières (car elle a déjà diffusé plusieurs expériences pilotes) au cours de l'année 2006.

Au total, voilà un type de projet qui mobilise beaucoup d'énergies et qui ne se réalise pas du jour au lendemain. À Miramichi, l'idée a connu plusieurs modifications au fil des ans, et c'est sans doute la complexité particulière de l'environnement régional qui explique, au moins en partie, les délais dans sa réalisation : le concept de départ, soit une radio modeste desservant essentiellement les 1500 francophones de la ville, ne rejoignait pas assez de gens pour le rendre viable. On a donc dû songer à un projet plus ambitieux, où les ondes du poste pourraient être captées dans les trois communautés francophones situées dans un rayon de quarante kilomètres de la ville de Miramichi, soit Rogersville au sud, Baie Sainte-Anne au sud-est et Neguac à l'est. Le bassin de population ainsi formé comprend environ 10000 personnes, ce qui représente un marché publicitaire beaucoup plus intéressant, puisque les radios communautaires, après avoir épuisé les subventions de départ, doivent s'autofinancer. Mais le hic, c'est que toute la programmation devra s'adapter à ce nouvel auditoire (les trois communautés étant très majoritairement francophones, à la différence des Acadiens très minoritaires à Miramichi) et, complication supplémentaire, les communautés cibles sont déjà desservies par d'autres radios communautaires, en place depuis longtemps : Neguac est dans la mire de CKRO, installée à Caraquet, et Rogersville et Baie Sainte-Anne sont dans le giron de Radio-Beauséjour, une très importante radio communautaire diffusant à partir de Shediac, dans le Sud-Est, et dont le signal est capté dans le nord du comté de 
Kent et dans le sud du comté de Northumberland grâce à de puissants émetteurs. Pour éviter des guerres interrégionales dont la petite communauté francophone de Miramichi ne pourrait que sortir perdante, il a fallu de laborieuses négociations avec les deux autres radios communautaires et avec les trois autres localités impliquées pour trouver une formule qui satisfasse à toutes ces parties. Au terme de ce long cheminement, les francophones de Miramichi espèrent que leur radio communautaire finira par ouvrir en 2006.

Si nous mentionnons ici le cas particulier de Miramichi, ce n'est pas du tout dans le but de critiquer cette communauté, ni de mettre en question le courage et la détermination de ses leaders et de ses bénévoles! Notre livre récent (Allain et Basque, 2005) regorge d'exemples de leur ténacité et de leur grande vaillance. C'est plutôt pour montrer que le centre scolaire-communautaire, qui constitue à notre avis un outil de développement incontournable, n'est quand même pas une recette magique : face à de grandes difficultés comme celles qui ont plu sur Miramichi et sa minorité francophone, son efficacité a été réduite. Le plus surprenant, c'est que malgré le contexte difficile, le Carrefour Beausoleil a su tirer son épingle du jeu et accumuler d'intéressantes réussites scolaires et communautaires, que ce soit par la reconnaissance de l'excellence scolaire de l'école, la programmation socioculturelle diversifiée, l'organisation d'activités sociales de toutes sortes et la création d'un certain nombre d'associations actives dans le milieu.

Justement, nous rejoignons ici le bilan des deux autres centres scolairescommunautaires du Nouveau-Brunswick: les trois centres ont été des bougies d'allumage dans leur environnement respectif. Ces centres sont issus de luttes parfois longues et houleuses pour obtenir des écoles françaises qui, lorsqu'elles ont été accordées l'ont été sous des modalités insatisfaisantes (vétusté et exiguïté des édifices scolaires, entre autres). Cet état de fait a ensuite cristallisé les revendications autour du concept de centre scolaire-communautaire. Une fois la formule acquise, à Fredericton en 1978, à SaintJean en 1984 et à Miramichi en 1986, la capacité de vitalisation communautaire des centres s'est rapidement affirmée. Retenons-en ici quatre illustrations : on se reportera à nos trois ouvrages pour trouver de nombreux exemples de chacune. La première, c'est le grand nombre et la diversité d'activités organisées après l'ouverture des centres, comparativement à ce qui existait auparavant. Le bénévolat est toujours un ingrédient crucial, mais la disponibilité d'un personnel communautaire embauché à plein temps, en animation et en communication, par exemple, ainsi que la présence d'infrastructures appropriées font une grande différence. Un petit exemple : le thêâtre amateur jouit d'une grande popularité en milieu francophone minoritaire. Les trois écoles disposent d'une jeune troupe étudiante, souvent encadrée par des employés communautaires, et ces troupes gagnent souvent des prix lors du Festival provincial de théâtre jeunesse. La présentation annuelle de leur pièce est un événement couru dans la communauté. De même, chez les adultes, chaque centre a sa troupe amateur ${ }^{12}$ qui fait salle comble lors des spectacles et qui rafle des prix, tour à tour, lors du Festival annuel de théâtre communautaire en Acadie, une manifestation qui existe depuis 1999. 
C'est dire aussi la diversité des organismes qui se sont développés, en grande partie depuis l'ouverture des trois centres : les données du tableau 1 soulignent la croissance du nombre des associations en vingt ans. Avant l'arrivée des centres, on trouvait à chaque endroit une poignée d'organismes; l'ouverture des centres a donné lieu à une véritable floraison de nouvelles associations, qui se sont diversifiées avec le temps : on retrouve aujourd'hui des groupes à vocation scolaire, culturelle, sportive, sociale, économique, et même politique, comme les sections locales de la Société des Acadiens et Acadiennes du Nouveau-Brunswick (SAANB). Comme on sait, les réseaux associatifs forts sont à la fois une condition et une manifestation de la vitalité communautaire (Allain, 2004, 2001; Allain et McKee-Allain, 2003).

Le troisième élément que nous voulons faire ressortir, c'est que les écoles faisant partie des centres sont des écoles de qualité. Elles offrent de l'avis de tous un excellent encadrement (le ratio professeur - élève y est plus favorable), et les enseignants, sentant qu'ils jouent un rôle clé dans le développement de la communauté, y sont souvent plus motivés que dans d'autres écoles. Malgré leur relative petite taille, comparativement par exemple à de plus grandes écoles des régions francophones ou aux écoles anglophones de leur milieu, les trois écoles rattachées aux centres excellent à plusieurs égards, que ce soit dans le domaine scolaire ou sportif. Dans certaines disciplines sportives, on remporte régulièrement des championnats régionaux ou provinciaux. Même chose lors de compétitions de musique, de mathématique ou de sciences (Expo-Sciences). Les élèves des trois écoles affichent souvent des résultats supérieurs lors des examens annuels du ministère de l'Éducation, et une proportion imposante de finissants et de finissantes (de la moitié aux trois quarts) choisissent de poursuivre des études postsecondaires, auxquelles ils réussissent très bien. Selon une étude récente effectuée à l'école SainteAnne à Fredericton, les élèves sont très fiers de leur école, et le sentiment d'appartenance y est élevé. Annie Pilote en explique l'importance :

Le sentiment d'appartenance à l'école française est particulièrement important en milieu minoritaire. À Fredericton, l'école est le principal milieu de vie francophone autre que la maison (bien que pour certains, l'école soit le seul endroit où le français occupe la place centrale). L'école procure à la langue française un milieu d'utilisation et une pertinence sociale. En plus d'offrir l'instruction en français, l'école permet aux jeunes de participer à diverses activités sportives, culturelles et sociales en français. Elle permet aussi de développer des relations sociales pleines de sens, dans lesquelles le français est un mode de communication possible et fréquent. Les finissants (12 ${ }^{\mathrm{e}}$ année) sont particulièrement conscients de cette réalité et plusieurs admettent avec regret qu'une fois diplômés, ils n'auront plus l'occasion de parler français aussi fréquemment. Dans ce sens, l'école joue un rôle déterminant dans la construction de l'identité des jeunes en milieu francophone minoritaire (2003: 42). 
L'excellence des écoles est reconnue dans divers classements récents. En mars 2003, un rapport du Atlantic Institute for Market Studies plaçait l'école Sainte-Anne de Fredericton au premier rang des écoles polyvalentes francophones de la province (Audas et Cirtwill, 2003) ${ }^{13}$. En avril 2004, c'était au tour de l'école du Carrefour Beausoleil de Miramichi de mériter la première place au classement du même organisme. Et en octobre 2004, le Bulletin des écoles secondaires de la province, préparé pour le compte du ministère de l'Éducation par le Fraser Institute, accordait le premier rang à l'école Samuel-de-Champlain de Saint-Jean, alors que l'école du Carrefour Beausoleil venait en deuxième place ${ }^{14}$. Évidemment, on pourrait toujours discuter de la validité de tels classements et des critères qu'ils utilisent, mais le fait que deux agences différentes attribuent de telles distinctions aux écoles des trois centres devrait signifier quelque chose $^{15}$. Nous estimons que la compétence et l'engagement des enseignants y sont pour beaucoup, de même parfois que les caractéristiques socioéconomiques des élèves (comme à Fredericton, où les enfants de fonctionnaires et de professionnels prédominent), sans oublier le ratio enseignant-élève avantageux. Comme l'avouait la directrice de l'école à Miramichi, parlant des 60 élèves se répartissant dans les quatre années du secondaire, "On a du temps à passer avec nos élèves. On connaît leurs forces et leurs faiblesses. On connaît aussi leur vécu» (citée dans Allain et Basque, $2005: 141,144$ ).

Une quatrième caractéristique des centres scolaires-communautaires néobrunswickois, c'est l'évolution semblable des communautés qu'ils desservent. Dans les trois cas, on peut distinguer quatre grandes étapes de leur cheminement, même si le moment et la durée de chacune peuvent varier selon le cas. On trouve d'abord une longue période de silence, voire de clandestinité, au cours de laquelle la communauté francophone minoritaire est dispersée au sein de l'ensemble urbain dont elle fait partie, n'a pas d'institutions propres (tels une école, une église, un centre culturel) ni d'associations pour défendre ses intérêts, bref, n'a pas de voix ni même d'existence comme communauté. Puis un certain réveil s'effectue; des leaders émergent; des revendications se définissent; un mouvement s'organise pour obtenir, d'abord, une école française, et par la suite, un centre scolaire-communautaire. Comme l'a montré Pilote (1999), la revendication et la création d'un centre scolaire-communautaire représentent un geste éminemment politique de la part de la communauté : c'est à la fois un acte d'affirmation et une démarche vers l'autonomie.

Le tableau 1 nous fournit l'information pour suivre ces deux premières étapes, la deuxième culminant avec l'ouverture des centres. La troisième phase porte sur la décennie suivant cette ouverture et se caractérise par l'organisation d'activités et de services diversifiés, tous offerts à l'intérieur des centres qui deviennent le lieu de rassemblement de la communauté et l'expression de sa vitalité. Enfin, vers le milieu des années 90 , on sent le besoin de sortir des centres pour certaines activités, devant la crainte d'une certaine ghettoïsation et le désir de rendre la communauté francophone plus visible à l'échelle de la région urbaine. Cela s'est effectué de diverses façons : la tenue hebdomadaire ou mensuelle de soirées sociales dans des bars du centre-ville avec des groupes musicaux francophones; les cérémonies annuelles du 15 août au centre-ville 
également (avec levée officielle du drapeau acadien et discours du maire de la ville, de politiciens provinciaux et fédéraux et des leaders de la communauté, sans oublier le traditionnel tintamarre et une fête populaire); l'organisation, à Saint-Jean en 1996 et à Fredericton en 2000, de la Finale des Jeux de l'Acadie, qui a, dans les deux cas, entraîné dans ces villes pendant plusieurs jours une affluence de quelques milliers de personnes (jeunes athlètes, entraîneurs et bénévoles locaux, parents et amis) qui se déplaçaient ici et là pour assister aux compétitions tenues sur des terrains d'institutions aussi bien anglophones que francophones et pour participer aux activités culturelles présentées dans le cadre des Jeux. Comme nous le montrons dans nos travaux (Allain et Basque, 2001, 2003; Allain, 2003), les Jeux de l'Acadie ont grandement contribué à faire connaître la communauté francophone auprès des anglophones et à leur montrer que les Acadiens pouvaient organiser avec succès de grands projets. Pour plusieurs anglophones que nous avons rencontrés, ce fut une révélation. Plusieurs de nos informateurs francophones nous ont aussi confié que c'est après la tenue réussie de la Finale des Jeux de l'Acadie, hautement médiatisée localement et à l'échelle de la province, qu'ils ont senti un changement d'attitude à leur endroit de la part des anglophones (entre autres chez les leaders municipaux et les gens d'affaires). Ceux-ci font preuve d'un plus grand respect à l'égard des francophones et d'une reconnaissance accrue de leur contribution au tissu social urbain et à la qualité de vie culturelle de la ville, sans compter les bénéfices économiques qu'ils ont réussi à obtenir pour la région, car les Jeux ont des retombées économiques importantes ${ }^{16}$.

On trouvera un indicateur de ce dernier aspect dans des analyses récentes de l'impact économique de la présence des francophones en milieu urbain, entreprises à l'initiative de la Société des Acadiens et Acadiennes du Nouveau-Brunswick et de ses sections locales. L'étude pour Miramichi, dévoilée en 2004, parle de retombées annuelles de l'ordre de 117 millions de dollars, en incluant les communautés francophones environnantes et les revenus générés par le tourisme québécois (LeBlanc, 2004). À SaintJean, l'étude d'impact rendue publique en mai 2004 estime les retombées économiques générées par les francophones à 90 millions de dollars, le tout à partir de données de 2001. Dans une conférence récente sur le sujet, le président de la Société des gens d'affaires et des professionnels francophones de Saint-Jean établit qu'en 2005 les retombées tournent probablement autour de 100 millions de dollars (Lacroix, 2005d). Dans la capitale provinciale de Fredericton, le même consultant établit l'impact économique annuel à 123 millions de dollars, et ce, comme pour Saint-Jean, en excluant les recettes engendrées par les touristes francophones (Lacroix, 2005d). En un mot, la francophonie est rentable! Voilà certes un facteur qui explique, de pair avec la reconnaissance de l'indéniable apport socioculturel de la communauté francophone à la diversité et à la qualité de vie de tous et de toutes, la plus grande ouverture dont font preuve les anglophones ces dernières années envers leurs concitoyens francophones.

Toutes ces caractéristiques communes n'empêchent pas certains centres de se démarquer de façon particulière en ayant en quelque sorte une "personnalité " bien à eux. Ainsi, nous avions noté, dans un texte antérieur (Allain, 2003 : 58-59), le caractère 
plus entrepreneurial de l'Association régionale de la communauté francophone de SaintJean (l'ARC-f de Saint-Jean) et de son leadership, responsables de la bonne marche du Centre scolaire-communautaire Samuel-de-Champlain, qui fêtait en 2005 le $20^{\mathrm{c}}$ anniversaire de son ouverture officielle. Ils furent en effet les premiers, en 1999, à fonder une Société de gens d'affaires et professionnels francophones et à entrer en partenariat avec deux organismes locaux de développement économique (Entreprise Saint John et le Bureau de commerce de Saint John) pour créer une organisation de promotion du bilinguisme appelée Avantage Saint John Advantage. Cette dernière a offert, par exemple, des ateliers de français le 23 février 2005 à une vingtaine de gens d'affaires, de chargés de projets et de cadres responsables des ressources humaines au sein de leur entreprise (Bouchard, 2005). Elle fait campagne pour promouvoir les réussites locales sur le plan du bilinguisme et œuvre pour la création de postes bilingues (LeBlanc, 2003 : 15). Le même organisme (auquel, faut-il encore le souligner, l'ARC-f de Saint-Jean participe de plain-pied, avec ses deux partenaires) a récemment eu l'idée de créer un site Internet pour loger le Bottin des services bilingues de la ville portuaire. Comme l'affirme le directeur général du Centre scolaire-communautaire, James Thériault, «Le bottin.org servira de pivot pour la promotion économique de la région de Saint-Jean et pourrait servir d'exemple pour d'autres communautés francophones en milieu minoritaire » (Roy, 2005).

Ajoutons un dernier élément : dans la foulée de la recherche, depuis le milieu des années 90, d'une plus grande visibilité et d'une plus forte présence au cœur du milieu urbain, l'ARC-f de Saint-Jean a tout récemment acheté un édifice patrimonial au centreville et vient d'y ouvrir, en 2005, un Centre culturel francophone (Pearn, 2005). Comme le Centre Samuel-de-Champlain est situé un peu à l'écart (on continuera d'y tenir les activités scolaires et une bonne partie des activités communautaires), il aura pour ainsi dire pignon sur rue au centre-ville, et on y présentera un certain nombre de prestations culturelles. En plus de loger quelques bureaux pour certains membres du personnel communautaire, l'édifice abritera également un bistro où francophones et francophiles pourront se côtoyer dans une ambiance française, en plein cœur de l'ancienne ville loyaliste!

En fait, deux des trois centres scolaires-communautaires, ceux de Fredericton et de Saint-Jean, ont depuis quelques années des problèmes d'espace, victimes jusqu'à un certain point de leur succès. Dans les deux cas, les inscriptions scolaires ont augmenté depuis quelques années, et des classes ont dû être ajoutées, empiétant sur l'espace réservé aux activités du secteur communautaire. À Fredericton, par exemple, l'école Sainte-Anne avait été construite en 1978 pour accueillir 400 élèves. En 1991, un agrandissement majeur permettait d'en desservir 700 (Lacroix, 2005b). En septembre 2005, on compte 1030 inscriptions! On a dû aménager les classes de $9^{\mathrm{c}}$ année dans quatre roulottes installées à l'arrière du Centre. La bibliothèque ne peut recevoir que deux classes à la fois, ce qui réduit la fréquence des visites. Le gymnase souffre de surcapacité, et 200 jeunes de la maternelle à la $2^{e}$ année se voient obligés d'utiliser un local temporaire ne pouvant accommoder que 20 élèves à la fois. Ceux de la $9^{\mathrm{e}}$ et de la $10^{\mathrm{e}}$ année, eux, doivent 
emprunter un autobus scolaire pour se rendre au gymnase de la University of New Brunswick. La cafétéria, de son côté, est conçue pour 260 élèves : on a dû diviser la période du dîner en quatre blocs de 20 minutes pour que tout le monde puisse y avoir accès (Lacroix, 2005b). Et on pourrait continuer : les enfants à besoins spéciaux, par exemple, manquent de locaux adéquats. La situation est devenue à ce point critique que le secteur communautaire a cédé tous les locaux qu'il pouvait à l'école (Mallet, 2005). Pour sa part, le Centre Samuel-de-Champlain à Saint-Jean vit une situation très semblable (classes logées dans des roulottes, etc.) ${ }^{17}$, et les francophones de la ville portuaire ont attendu eux aussi avec impatience l'annonce officielle d'un agrandissement important. Enfin, en novembre 2005, les gouvernements fédéral et provincial annonçaient le renouvellement de trois ententes en matière d'éducation au montant de 610 millions de dollars, dont 28 millions de dollars pour des agrandissements aux centres scolaires-communautaires de Fredericton et de Saint-Jean (Gagnon, 2006).

\section{Les centres scolaires-communautaires au Canada}

À l'heure actuelle, il existe 22 centres scolaires-communautaires au Canada, et quelques autres sont en préparation. La formule est populaire et fait consensus (Thériault, 2003 : 52), mais on la connaît relativement peu, puisque les chercheurs n'y ont pas consacré beaucoup d'études (Gérin-Lajoie, 2003 : 110). Parmi les exceptions, mis à part nos trois ouvrages, on compte les travaux d'Annie Pilote $(1999,2003)$ sur l'école Sainte-Anne à Fredericton, et l'analyse de sept modèles de gestion relevés par Renée Delorme et Yvonne Hébert (1998) dans les centres scolaires-communautaires au Canada. Malgré la pénurie relative de recherches sur le sujet, un fait se dégage : la clé du succès de ces centres semble reposer sur un partenariat réel entre l'école et le centre communautaire (Bisson, 2003; Hébert, 2003 : 224).

Dans cette section, nous allons comparer certaines caractéristiques des trois centres du Nouveau-Brunswick avec celles des 18 autres centres situés ailleurs au Canada. Il ne s'agit bien sûr que d'une esquisse préliminaire, qui aura à être approfondie, et surtout, contextualisée ${ }^{18}$.

Le tableau 2 (voir la colonne Total) permet de confirmer ce que nous savions d'un centre scolaire-communautaire: selon une compilation récente (Poirier, LeBlanc et Thériault, 2003), la grande majorité des 21 centres alors en existence au Canada, soit plus de 80 p. 100 d'entre eux, disposent entre autres d'un gymnase ${ }^{19}$, d'une bibliothèque scolaire et communautaire, d'une cafétéria, et plus de 70 p. 100 ont une salle de spectacle et un centre de ressources communautaires. L'accès aux locaux est gratuit pour les organismes de la communauté. Plus de la moitié ont une galerie d'art, une salle de musique, et environ le tiers des centres abritent une garderie et une station de radio. Bien sûr, en plus de l'école, la quasi-totalité des centres hébergent une ou plusieurs maternelles ainsi qu'une ou des classes de prématernelle, mais seulement le tiers offrent des services religieux. 
Tableau 2

Services et activités offerts par les centres scolaires-communautaire (CSC) : une comparaison Nouveau-Brunswick-Canada

\begin{tabular}{|c|c|c|c|}
\hline Services & $\begin{array}{c}3 \text { CSC du Nouveau- } \\
\text { Brunswick }\end{array}$ & $\begin{array}{c}18 \text { CSC ailleurs au } \\
\text { Canada }^{1}\end{array}$ & Total \\
\hline Bureaux pour les organismes & 2 & 17 & 19 \\
\hline Accès aux locaux sans frais & 3 & 15 & 18 \\
\hline Services de secrétariat & 3 & 8 & 11 \\
\hline $\begin{array}{l}\text { Centre de ressources } \\
\text { communautaires }\end{array}$ & 1 & 14 & 15 \\
\hline $\begin{array}{l}\text { Accès ordinateur/télécopieur/ } \\
\text { photocopie }\end{array}$ & 3 & 9 & 12 \\
\hline Bibliothèque publique & 3 & 10 & 13 \\
\hline Cafétéria/cuisine & 3 & 15 & 18 \\
\hline Maternelle & 3 & 15 & 18 \\
\hline Gymnase & 3 & 14 & 17 \\
\hline Prématernelle & 3 & 15 & 18 \\
\hline Théâtre/salle de spectacle & 3 & 12 & 15 \\
\hline Galerie d'art & 3 & 8 & 11 \\
\hline Salle de musique & 3 & 10 & 13 \\
\hline Salle/station de radio & 2 & 5 & 7 \\
\hline Services religieux & 2 & 5 & 7 \\
\hline Garderies & 3 & 5 & 8 \\
\hline \multicolumn{4}{|c|}{ Activités socioculturelles pour adultes } \\
\hline Sport (gymnase) & 3 & 17 & 20 \\
\hline Spectacles & 3 & 16 & 19 \\
\hline Programmation artistique & 3 & 16 & 19 \\
\hline Formation & 3 & 16 & 19 \\
\hline Soirées sociales & 3 & 17 & 20 \\
\hline Théâtre communautaire & 3 & 11 & 14 \\
\hline Activités/clubs sociaux & 3 & 15 & 18 \\
\hline \multicolumn{4}{|c|}{ Activités socioculturelles et services aux étudiants } \\
\hline Bibliothèque scolaire & 3 & 15 & 18 \\
\hline Sports et gymnase & 3 & 16 & 19 \\
\hline Spectacles culturels & 3 & 16 & 19 \\
\hline Scouts/guides & 2 & 7 & 9 \\
\hline Troupe de thêâtre & 3 & 13 & 16 \\
\hline Chorale/orchestre & 3 & 9 & 12 \\
\hline Maison des jeunes (camp d'été) & 3 & 9 & 12 \\
\hline Radio étudiante & 0 & 3 & 3 \\
\hline
\end{tabular}

Source : Poirier, LeBlanc et Thériault, 2003 : 59-60; calculs de l'auteur.

Note :

1. Un $22^{\circ}$ CSC a ouvert ses portes en avril 2005 à Saint-Jean, Terre-Neuve-et-Labrador. 
Sur le plan des activités socioculturelles pour les adultes, la vaste majorité (plus de 85 p. 100 des centres) offre une programmation sportive, culturelle et sociale, ainsi que des cours, et les deux tiers présentent du théâtre communautaire. Pour les élèves, en plus de la bibliothèque et du gymnase, presque tous les centres offrent des activités culturelles et, fait à noter, plus des trois quarts ont une troupe de théâtre scolaire. Près de 60 p. 100 comptent une chorale ou un orchestre et organisent un camp d'été pour les jeunes. Moins de la moitié des centres ont une ou des troupes de scouts ou de guides, encore que notre expérience de terrain indique que ces groupes sont souvent comptabilisés avec les activités paroissiales, menant peut-être ici à une sous-représentation du phénomène. Enfin, très peu d'écoles, soit seulement 3 sur 21 , semblent disposer d'une radio étudiante, probablement en raison du coût de l'équipement technique requis.

Voilà donc pour l'éventail des installations et des services offerts à l'échelle canadienne par les centres scolaires-communautaires. Si nous comparons maintenant les trois centres du Nouveau-Brunswick (colonne 1 du tableau 2) avec les 18 autres ailleurs au pays (colonne 2), les données indiquent une plus grande complétude institutionnelle (Breton, 1964) des premiers par rapport aux seconds. En effet, pour la plupart des éléments, soit 25 sur 31 , les centres néo-brunswickois affichent une cote parfaite de 100 p. 100, ce qui n'est jamais le cas pour les autres: les résultats les plus élevés de ces derniers atteignent 94 p. 100 (17 sur 18) dans seulement trois catégories, puis 89 p. 100 dans cinq autres, et 83 p. 100 dans six autres.

Quels facteurs pourraient expliquer ces variations? À première vue, il pourrait simplement s'agir de la taille du centre. Par exemple, si nous considérons le nombre d'élèves inscrits aux écoles (tableau 3), nous constatons que c'est au Nouveau-Brunswick que la moyenne est la plus élevée, avec 569 élèves par établissement, suivi de l'Ontario avec 336,3 et de la Nouvelle-Écosse avec 228,5. Terre-Neuve-et-Labrador figure en dernière place avec 91 élèves, mais ce total devrait augmenter avec l'ouverture récente, en avril 2005, d'un second centre scolaire-communautaire, cette fois à St. John's. Évidemment, des moyennes peuvent être trompeuses : ainsi, au Nouveau-Brunswick, les trois centres ont une population étudiante respective de 1000 à Fredericton, de 500 à Saint-Jean et de 230 à Miramichi. Mais en gros, cette mesure nous donne une indication de la taille relative des centres, d'autant plus qu'elle est confirmée par l'examen de la taille moyenne des complexes : les trois centres néo-brunswickois ont une superficie moyenne de 11000 mètres carrés, alors que les dix-huit autres centres au Canada recouvrent une superficie moyenne de 4600 mètres carrés (calculé à partir de Poirier, LeBlanc et Thériault, $2003: 57-58$ ). À défaut donc de mesures plus précises, à l'échelle canadienne, des effectifs enseignants et communautaires, ainsi que de la participation des francophones aux activités organisées dans les centres, il faudra donc se contenter ici des indicateurs évoqués tantôt (les statistiques du tableau 2, plus le nombre d'élèves et la superficie des centres) pour estimer le type d'installations retrouvées dans les centres et la gamme de services et d'activités qui y sont offerts ${ }^{20}$. 
Tableau 3

Nombre de centres scolaires-communautaires (CSC) par province et effectifs scolaires (2003)

\begin{tabular}{lccr}
\hline \multicolumn{1}{c}{ Provinces } & Nombre de CSC & Nombre d'élèves & $\begin{array}{c}\text { Nombre moyen d'élèves } \\
\text { en CSC }\end{array}$ \\
\hline Terre-Neuve-et- & & & \\
Labrador & 1 & 91 & 91 \\
Nouvelle-Écosse & 2 & 457 & 228,5 \\
Nouveau-Brunswick & 3 & 1707 & 569 \\
Île-du-Prince-Édouard & 3 & 603 & 201 \\
Ontario & 3 & 1009 & 336,3 \\
Saskatchewan & 2 & 334 & 167 \\
Alberta & 6 & 1078 & 69,7 \\
Colombie-Britannique & 1 & 60 & \\
& & & 254,2 \\
\hline Total & 21 & 5339 & \\
\hline
\end{tabular}

Source : Poirier, LeBlanc et Thériault, 2003; calculs de l'auteur.

Tableau 4

Trois vagues de création de centres scolaires-communautaires (CSC)

\begin{tabular}{rl}
\hline Périodes & \multicolumn{1}{c}{ Nombre de CSC } \\
\hline $1978-1986$ & 3 (au Nouveau-Brunswick) \\
$1991-1996$ & 11 (dont 4 en Atlantique) \\
$1997-2005$ & 8 (dont 3 en Atlantique) \\
& 22 \\
\hline Total (2005) & \\
\hline
\end{tabular}

Source : Poirier, LeBlanc et Thériault, 2003 : 57-58.

Tableau 5

Répartition régionale des centres scolaires-communautaires (CSC)

\begin{tabular}{lc}
\hline Régions & Nombre de CSC \\
\hline Atlantique & 10 \\
Ontario & 3 \\
Ouest & 9 \\
Total (2005) & 22 \\
\hline
\end{tabular}

Source : Poirier, LeBlanc et Thériault, 2003 : 57-58. 


\section{Conclusion}

Ce qui nous intéresse ici, au fond, va bien au-delà des typologies que l'on pourrait dresser à partir de ces mesures. L'objectif poursuivi, c'est la connaissance et la compréhension de la vitalité communautaire effective au sein des francophonies canadiennes minoritaires. Nous avons pu l'établir d'assez près pour les trois communautés acadiennes que nous avons étudiées plus en profondeur dans nos travaux récents ${ }^{21}$. D'autres études qualitatives, même si elles étaient moins exhaustives, dans d'autres régions du Canada, nous permettraient de cerner le phénomène avec plus de précision. Nos travaux s'inscrivent d'ailleurs dans la mouvance d'un bon nombre de recherches en cours sur la vitalité sociolinguistique des communautés francophones minoritaires, que ce soit au sein de centres (comme le Centre interdisciplinaire de recherche sur la citoyenneté et les minorités, le CIRCEM, à l'Université d'Ottawa) ou d'instituts (tel l'Institut canadien de recherche sur les minorités linguistiques, l'ICRML, à l'Université de Moncton), ou à l'intérieur de ministères fédéraux comme celui du Patrimoine canadien (voir O'Keefe, 2001; Floch et Frenette, 2005; Johnson et Doucet, 2005). Il y a sur ce plan une importante convergence d'intérêt et de préoccupations, notamment en raison du débat récurrent sur l'assimilation linguistique et l'avenir des communautés francophones à l'extérieur du Québec. Les enjeux autour de cette question sont de taille, comme on sait, et ils sont autant d'ordre sociologique que politique (voir par exemple Allain, 2003 : 527-528; Allain et McKee-Allain, 2003 : 539-541).

Nous vivons à une époque remplie de commémorations : l'Acadie a fêté son $400^{\circ}$ en 2004, le Québec s'apprête à faire de même en 2008. S'il est important de souligner les dates historiques marquantes d'une collectivité, il est tout aussi important, sinon plus, d'en étudier la vitalité actuelle, ses manifestations et ses causes: en ce sens, pour paraphraser Joseph Yvon Thériault (2004), ce qui nous intéresse d'abord, au-delà de leur histoire particulière, c'est l'aspect "moderne, urbain, voire cosmopolite" des communautés francophones minoritaires (voir Allain, 2005b : 124-127). C'est ce à quoi s'emploient nos travaux.

\section{NOTES}

1. Une suite heureuse au colloque de mai 2004 à l'ACFAS, qui portait sur "La vitalité des communautés francophones du Canada : si destinée n'était pas synonyme de densité ". Voir notre communication à ce colloque: Allain (2005a).

2. Parmi les précurseurs du concept, mentionnons Ignacy Sachs en France et ses travaux sur l'écodéveloppement au cours des années 70 (voir son livre-synthèse publié en 1980), Ernest F. Schumacher (1973) et son idée de technologies appropriées, ainsi que Lester Brown, le fondateur du Worldwatch Institute, et auteur d'un livre emblématique en 1981. 


\section{Allain}

3. Voir entre autres Mormont, 2002. Un rapport de la Banque mondiale recensait, dès la fin des années 80 , pas moins de 37 acceptions différentes du développement durable, un autre auteur en comptait soixante, et le Rapport Brundtland lui-même en inclurait six (Latouche, $2004: 53$ ).

4. À moins qu'elle ne soit un passe-partout, auquel cas elle peut à l'occasion avoir son utilité. Mais sur le plan conceptuel, peuton vraiment se fier à une formule passe-partout?

5. Sans compter la question non résolue de ce qui devrait être durable, le système actuel de production ou une alternative fondamentalement différente (Mormont, $2002: 13$ ).

6. Voir Allain et Basque (2001), Allain (2002), et Allain et Basque (2003, 2005), ainsi que la synthèse des deux premières recherches dans Allain (2003). Sur la vitalité des trois communautés étudiées, voir Allain (2005a).

7. Nous rejoignons ainsi par exemple les conclusions des travaux de Aunger (2002), Cardinal et McKee-Allain (1999), Couture (2001), Gilbert (2004), O’Keefe (2001), Stebbins (2000) et Thériault (1995, 1999).

8. À noter en passant que l'usage du terme " hors Québec" pour désigner les francophonies canadiennes minoritaires est un terme périmé depuis le début des années 90. C'est pour cette raison que l'ancienne Fédération des francophones hors Québec changeait de nom en 1991 pour devenir la Fédération des communautés francophones et acadienne du Canada.

9. En attendant que la communauté se dote de sa propre église, comme les francophones de Saint-Jean l'ont fait en 1999, et ceux de Fredericton, en 2000.

10. Les trois communautés étudiées disposent d'outils communicationnels professionnels et efficaces, dont des bulletins d'information largement diffusés dans la communauté : à Saint-Jean, c'est entre autres Le Saint-Jeannois, à Fredericton, L'Info-Lien, et à Miramichi, Le Réveil.

11. Il faut dire qu'avant toutes ces fermetures, en plus d'être déjà de petite taille, la communauté francophone était fragmentée (pour ne pas dire balkanisée, selon l'expression d'un consultant : Poulin, 1992: 55) en trois blocs : au Recensement de 1986, les 2430 personnes de langue maternelle française de Chatham et Newcastle (les deux villes fusionnées en 2000 par le gouvernement provincial pour donner naissance à la ville de Miramichi) comprenaient les 700 Québécois de la base militaire, les 400 habitants de Beaverbrook et de Bellefond, deux petites localités à une quinzaine de kilomètres au nord de Miramichi (Allain et Basque, 2005 : 207-209), et environ 1400 Acadiens vivant à Chatham et à Newcastle.

12. La troupe de Fredericton s'appelle Les fous de la scène, celle de Miramichi, la Troupe Lalarmaloeil, et celle de Saint-Jean, Le théâtre du Trémolo.

13. L'école figurait au deuxième rang des 71 écoles polyvalentes de la province, tout juste derrière la Sir James Dunn Academy de St. Andrews.

14. Voir l'article intitulé "Les centres scolaires communautaires dominent le classement 2004 ", dans " Le Bulletin des écoles secondaires 2004 ", L'Acadie Nouvelle, cahier spécial, 23 octobre 2004, p. 3.

15. Malgré leur insertion particulière dans la communauté et leurs retombées dépassant largement le plan scolaire, précisons que les écoles des centres scolaires-communautaires sont aussi de "vraies " écoles, et non pas des exemples de ce que Thériault (2003) appelle "l'école d'en bas ", c'est-à-dire une école asservie à la communauté et offrant " une pédagogie de l'exiguïté, une pédagogie du minoritaire " qui réduit la culture au local, par opposition à l'école de la nation (l'école d'en haut), qui ouvre l'élève à des horizons plus vastes. En fait, les écoles des trois centres scolaires-communautaires du NouveauBrunswick donnent une formation basée sur des standards universels à travers leurs programmes, mais sans oublier, notamment par l'intermédiaire des activités parascolaires, leur insertion dans une communauté spécifique, tant historique qu'actuelle. Nous reconnaissons évidemment qu' "en milieu minoritaire, les objectifs de l'éducation de langue française dépassent nettement ceux assignés dans un milieu majoritaire ", et que la mission de l'école francophone dans de tels contextes " comprend non seulement les savoir $[$ sic $]$, les savoir-faire et les savoir-être, mais [...] les savoir-agir, les savoir-vivre ensemble et les savoir-devenir nécessaires à la préparation des gens qui bâtiront la communauté " (Gilbert, LeTouzé, Thériault et Landry, 2004 : 9). Mais l'école minoritaire doit aussi former à l'universel.

16. Pour une analyse globale du phénomène des Jeux de l'Acadie, voir Allain (sous presse).

17. La garderie à elle seule comptait, en 2004-2005, 225 enfants de 1 à 12 ans et un personnel de 28 éducatrices.

18. Entre autres par des études de communauté : il importe de savoir, par exemple, que certaines minorités francophones urbaines sont de plus en plus hétérogènes, mais qu'elles sont également fragmentées et divisées, comme dans le cas analysé par Hébert et Buteau (2003).

19. Par définition, évidemment, tous les centres comprennent une école.

20. Le tableau 4 indique pour sa part que la création des centres scolaires-communautaires s'est faite en trois vagues successives, les trois premiers étant établis au Nouveau-Brunswick entre 1978 et 1984 . Onze autres centres, dont quatre en Atlantique, sont mis sur pied entre 1991 et 1996. Et depuis 1997, huit seront ouverts, dont trois autres en Atlantique. C'est sans doute ce qui explique que la région Atlantique soit dotée du plus grand nombre de centres, soit dix (voir tableau 5), suivie tout de même de près par l'Ouest canadien, avec neuf; l'Ontario en compterait trois. Par ailleurs, parmi les trois projets en développement, deux sont situées dans l'Ouest et un en Ontario.

21. Voir Allain et Basque, 2001, 2003, 2005; Allain et McKee-Allain, 2003; Allain, 2002, 2003, 2004, 2005a, 2006. 


\section{BIBLIOGRAPHIE}

ALlAIN, Greg (2001), "La société acadienne en réseaux : trois études de cas dans les domaines du sport, des affaires et de l'Acadie "mondiale" ", Revue de l'Université de Moncton, numéro hors série, p. 191-204.

ALLAIN, Greg (2002), "La communauté francophone de Saint-Jean, Nouveau-Brunswick : de la survivance à l'affirmation ", Francophonies d'Amérique, $\mathrm{n}^{\circ} 14$ (automne), p. 37-53.

ALLAIN, Greg (2003), "Les études de communautés en milieu francophone urbain minoritaire : les cas de Saint-Jean et de Fredericton ", Francophonies d'Amérique, nº 16 (automne), p. 45-65.

ALLAIN, Greg (2004), "Fragmentation ou vitalité? Regard sociologique sur l'Acadie actuelle et ses réseaux associatifs ", dans Simon Langlois et Jocelyn Létourneau (dir.), Aspects de la nouvelle francophonie canadienne, Québec, Presses de l'Université Laval, p. 231-254.

ALlAIN, Greg (2005a), "Les conditions de la vitalité socioculturelle chez les minorités francophones en milieu urbain : deux cas en Acadie du Nouveau-Brunswick ", Francophonies d'Amérique, n 20 (automne), p. 133-146.

Allain, Greg (2005b), "Les sociologues et l'Acadie : l'évolution des regards sociologiques sur la société acadienne ", dans Marie-Linda Lord (dir.), L'émergence et la reconnaissance des études acadiennes : à la rencontre de Soi et de l'Autre, Moncton, Association internationale des études acadiennes, p. 113-136.

AllaiN, Greg (2006), "Une innovation organisationnelle acadienne pour les minorités francophones en milieu urbain : le Centre scolaire-communautaire en Acadie du Nouveau-Brunswick et son essaimage ailleurs au Canada ", dans André Magord (dir.), Adaptation et innovation : expériences acadiennes contemporaines, Bruxelles, Éditions Peter Lang, p. 141-154.

ALlaIN, Greg (sous presse), " Genèse, structure et bilan d'une manifestation sportive et identitaire pour la jeunesse acadienne : les Jeux de l'Acadie dans les provinces maritimes du Canada ", dans Jean-Pierre Augustin et Christine Dallaire (dir.), Jeux, sports et francophonie : l'exemple du Canada, Talence, Éditions de la Maison des sciences de l'homme d'Acquitaine.

ALLAIN, Greg, et Maurice BASQUE (2001), De la survivance à l'effervescence : portrait historique et sociologique de la communauté francophone et acadienne de Saint-Jean, Nouveau-Brunswick, Association régionale de la communauté francophone de SaintJean.

ALLAIN, Greg, et Maurice BASQUE (2003), Une présence qui s'affirme : la communauté acadienne et francophone de Fredericton, Nouveau-Brunswick, Moncton, Éditions de la Francophonie.

AllaIN, Greg, et Maurice BASQUE (2005), Du silence au réveil: la communauté acadienne et francophone de Miramichi, Nouveau-Brunswick, Miramichi, Centre communautaire Beausoleil.

Allain, Greg, et Isabelle MCKeE-Allain (2003), "La société acadienne en l'an 2000 : identité, pluralité et réseaux ", dans André Magord (dir.), L'Acadie plurielle : dynamiques identitaires collectives et développement au sein des réalités acadiennes, avec la collaboration de Maurice Basque et d'Amélie Giroux, Poitiers, Institut d'études acadiennes et québécoises; Moncton, Centre d'études acadiennes, p. 535-565.

Audas, Rick, et Charles CirTwill (2003), Grading our Future: Atlantic Canada's High Schools' Accountability and Performance in Context, Halifax, Atlantic Institute for Market Studies.

AUNGER, Edmund (2002), "Obsèques prématurées: la disparition des minorités francophones et autres illusions nationalistes ", Revue d'études constitutionnelles = Review of Constitutional Studies, vol. 7, n² 2, p. 120-142.

"Avec un pouvoir d'achat annuel de $\$ 123$ millions : la communauté francophone est devenue un pilier économique et social " (2005), La voix des affaires, vol. 3, $\mathrm{n}^{\circ} 2$ (avril), p. 18.

BABIN, Ronald (2004), "La modernisation sociétale comme application progressiste du développement durable ", dans Louis Guay et al. (dir.), Les enjeux et les défis du développement durable : connâttre, décider, agir, Québec, Presses de l'Université Laval, p. 79-101.

BERNARD, Roger (1990), Le déclin d'une culture: recherche, analyse et bibliographie: Francophonie hors Québec 1980-1989, livre 1 : Vision d'avenir, Ottawa, Fédération des jeunes Canadiens français.

BERNARD, Roger (1996), De Québécois à Ontarois, Ottawa, Le Nordir.

BERNARD, Roger (1998), Le Canada français : entre mythe et utopie, Ottawa, Le Nordir.

Bisson, Ronald (2003), Étude sur les conditions de succès des centres scolaires et communautaires: rapport final, Ottawa, Patrimoine Canadien.

Bouchard, Marc-André (2005), "Des ateliers de français offerts aux Saint Johners ", Le Saint-Jeannois, n 66, 19 mars, p. 2. BRETON, Raymond (1964), "Institutional Completeness of Ethnic Communities and the Personal Relations of Immigrants ", American Journal of Sociology, vol. 70 (juillet), p. 193-205.

BRETON, Raymond (1983), “La communauté ethnique, communauté politique ", Sociologie et sociétés, vol. 15, $\mathrm{n}^{\circ} 2$ (octobre), p. 23-37. 
BRETON, Raymond (1984), "Les institutions et les réseaux d'organisation des communautés ethnoculturelles ", État de la recherche sur les communautés francophones hors Québec, actes du premier colloque national des chercheurs, des 9, 10 et 11 novembre 1984, Ottawa, Fédération des francophones hors-Québec, p. 4-20.

BROWN, Lester (1981), Building a Sustainable Society, New York, W. W. Norton.

CARDinal, Linda, et Isabelle MCKeE-Allain (1999), "Enjeux et défis de la recherche féministe en milieu francophone minoritaire au Canada ", dans Huguette Dagenais (dir.), La recherche féministe dans la francophonie : pluralité et convergences, Montréal, Éditions du remue-ménage, p. 449-465.

CASTONGuAy, Charles (1993), "Le déclin des populations francophones de l'Ouest canadien ", Cahiers franco-canadiens de l'Ouest, vol. 5, n" 2, p. 147-153.

Castonguay, Charles (1994), "Évolution récente de l'assimilation linguistique au Canada ", dans Claude Poirier (dir.), Langue, espace et société : les variétés du français en Amérique du Nord, Sainte-Foy, Presses de l'Université Laval, p. 277-311.

CASTONGuay, Charles (1996), "Évolution de l'anglicisation des francophones au Nouveau-Brunswick, 1971-1991", dans Lise Dubois et Annette Boudreau (dir.), Les Acadiens et leurs langues : quand le français est minoritaire, Moncton, Éditions d'Acadie et le Centre de recherche en linguistique appliquée, p. 47-62.

CASTONGuaY, Charles (2002), "Assimilation linguistique et remplacement des générations francophones et anglophones au Québec et au Canada ", Recherches sociographiques, vol. 43, n"1 (janvier-avril), p. 149-182.

"Les centres scolaires communautaires dominent le classement 2004" (2004), dans "Le Bulletin des écoles secondaires 2004 ", L'Acadie Nouvelle, cahier spécial, 23 octobre, p. 3.

COLETTE, Lucille (2003), "Renouvellement du concept des centres scolaires-communautaires: plan d'action pour le renouvellement du concept des centres scolaires-communautaires au Nouveau-Brunswick, dans le cadre de la prochaine entente fédérale-provinciale sur les langues officielles ", Moncton, novembre.

Courure, Claude (2001), "La disparition inévitable des francophones à l'extérieur du Québec : un fait inéluctable ou le reflet d'un discours déterministe? ", Francophonies d'Amérique, n"11, p. 7-18.

DAlY, Herman (1990), "Towards Some Operational Principles of Sustainable Development ", Ecological Economics, n 2, p. 1-6.

Delorme, Renée, et Yvonne HÉBERT (1998), "Une analyse critique de sept modèles de gestion de centres scolairescommunautaires ", dans Georges Duquette et Pierre Riopel (dir.), L'éducation en milieu minoritaire et la formation des maitres en Acadie et dans les communautés francophones du Canada, Sudbury, Presses de l'Université Laurentienne, p. 199-230.

"L'enseignement au Nouveau-Brunswick " (2004), Vivre à l'étranger, hors-série, n" 2 (décembre), p. 11-12.

FlOCH, William, et Yves FRENETTE (2005), Vitalité des communautés, confiance des communautés : forum de recherche sur les langues officielles, Ottawa, Patrimoine Canada et Réseau de la recherche sur la francophonie canadienne.

GAGNON, Charles-Antoine (2006), "Début des travaux à l'école Sainte-Anne ", L'Acadie Nouvelle, 25 mai, p. 5.

Gendron, Corinne (2004), "Le développement durable : un nouvel enjeu de l'historicité ", dans Louis Guay et al. (dir.), Les enjeux et les défis du développement durable : connaître, décider, agir, Québec, Presses de l'Université Laval, p. 59-78.

GÉRIN-L.AjOIE, Diane (2003), Parcours identitaires de jeunes francophones en milieu minoritaire, Sudbury, Prise de Parole.

GIl.BERT, Anne (2004), "Les minorités francophones du Canada : milieux de vie et identité ", dans Didier Terrier et Tamara Kondratieva (dir.), Territoire, frontières, identités : concordances et discordances, Lille, Revue du Nord, n ${ }^{\circ}$ 18, p. 171-181.

GILBERT, Anne, Sophie LeTouzé, Joseph Yvon ThÉRIAult et Rodrigue LANDry (2004), Le personnel enseignant face aux défis de l'enseignement en milieu minoritaire francophone, Ottawa, CIRCEM - Patrimoine canadien, (septembre).

GuaY, Louis, et al. (dir.) (2004), Les enjeux et les défis du développement durable : connaître, décider, agir, Québec, Presses de l'Université Laval.

HÉBERT, Yvonne (2003), "Changement de frontières identitaires et citoyennes: vers un programme de recherche sur l'enseignement et l'apprentissage des sciences humaines en milieu francophone minoritaire ", dans Réal Allard (dir.), Actes $d u$ colloque pancanadien sur la recherche en éducation en milieu francophone minoritaire: bilan et prospectives, Moncton, Centre de recherche et développement en éducation; Québec, Association canadienne d'éducation de langue française, p. 216-230.

HÉBERT, Yvonne, et Daniel ButEau (2003), “École, spatialité et communauté : perspective historique de l'évolution de la francophonie calgaréenne ", dans Nathalie Kermoal (dir.), Variations sur un thème: la francophonie albertaine dans tous ses états, Edmonton, Salon d'histoire de la francophonie albertaine, p. 153-176.

Johnson, Marc, et Paule Doucet (2005), "Renforcer les bases: forum de discussion sur la vitalité des communautés de langue officielle en milieu minoritaire ", document de réflexion, Ottawa, Commissariat aux langues officielles, (septembre).

JoY, Richard (1967), Languages in Conflict: The Canadian Experience, Ottawa, l'auteur.

LACRoIX, Isabelle (2005a), " Des parents se mobilisent pour l'école Sainte-Anne ", L'Acadie Nouvelle, 27 mai, p. 9.

LACROIX, Isabelle (2005b), "L'école Sainte-Anne victime de son succès : l'établissement de Fredericton manque d'espace pour accueillir ses 1000 élèves ", L'Acadie Nouvelle, 18 mai, p. 4.

LACROIX, Isabelle (2005c), "Fredericton : les gens d'affaires francophones veulent fonder une association ", L'Acadie Nouvelle, 21 avril, p. 8.

LACROIX, Isabelle (2005d), "Les francophones ont un plus grand impact économique à Saint-Jean ", L'Acadie Nouvelle, 28 mars. 


\section{Une nouvelle ressource collective pour des communautés francophones}

LATOUCHE, Serge (2004), Survivre au développement : de la décolonisation de l'imaginaire économique à la construction d'une société alternative, Paris, Mille et une nuits.

LEBLANC, Marc (2004), Retombées économiques des francophones dans le Miramichi, 19 février.

LEBlanc, Mireille E. (2003), "St-Jean : un pour tous et tous pour un ", Le journal de commerce de l'Atlantique, vol. 3, n 3 (mai-juin), p. 13-19.

LÉGĖRE, Clarence (1989), "Les centres scolaires et communautaires : outils de développement ", Éducation et francophonie, vol. 17, nº 3 (décembre), p. 29-32.

Mallet, Geneviève (2005), "Dossier agrandissement: l'annonce se fait toujours attendre ", L'Info-Lien, vol. 17, $\mathrm{n}^{\circ} 7$ (septembre), p. 1.

MEadows, Donella H. et al. (dir.) (1972), The Limits to Growth: A Report for the Club of Rome's Project on the Predicament of Mankind, New York, Universe Books.

Mormont, Marc (2002), "Préface : du concept au mode d'emploi ", dans Edwin Zaccaï (dir.), Le développement durable : dynamique et constitution d'un projet, Bruxelles, Presses interuniversitaires européennes, Éditions Peter Lang, p. 9-16.

O'KEEFE, Michael (2001), Minorités francophones: assimilation et vitalité des communautés, $2^{c}$ édition, Ottawa, Patrimoine canadien.

PARIZEAU, Marie-Hélène (2004), « Du développement au développement durable : l'environnement en plus. Analyse éthique et politique ", dans Louis Guay et al. (dir.), Les enjeux et les défis du développement durable : connaître, décider, agir, Québec, Presses de l'Université Laval, p. 305-326.

PEARN, Matt (2005), "Francophones go from Survival to Success ", Telegraph-Journal, $1^{\text {ex }}$ octobre, p. B5.

Pelletier, Réjean (2003), "Un divorce consommé ", dans Simon Langlois et Jean-Louis Roy (dir.), Briser les solitudes: les francophonies canadiennes et québécoises, Québec, Éditions Nota bene, p. 31-42.

PILOTE, Annie (1999), "L'analyse politique des centres scolaires et communautaires en milieu francophone minoritaire ", Education et francophonie, vol. 27, $\mathrm{n}^{\circ} 1$ (printemps), p. 1-13.

Pilote, Annie (2003), "Sentiment d'appartenance et construction de l'identité chez les jeunes fréquentant l'école Sainte-Anne en milieu francophone minoritaire ", Francophonies d'Amérique, n' 16 (automne), p. 37-44.

Poirier, Bernard, Conrad LeBlanc et Paul-Émile Thériault (2003), Les phares communautaires et scolaires du Canada, Fredericton, Centre communautaire Sainte-Anne.

Poulin, Pierre (1992), "Le Centre scolaire-communautaire Beausoleil ", Evaluation de l'entente auxiliaire Canada/NouveauBrunswick sur les centres scolaires-communautaires, rapport final, 9 novembre, p. 48-96.

Report of the World Commission on the Environment and Development, "Our Common Future" (Bruntland Report) (1987), Oxford, Oxford University Press; traduction française, 1989, Notre avenir à tous, Montréal, Éditions du Fleuve.

ROY, Martin (2005), " Nouveau site Internet pour promouvoir les services bilingues à Saint-Jean ", L'Acadie Nouvelle, 31 mars, p. 11.

SACHS, Ignacy (1980), Stratégies de l'écodéveloppement, Paris, Éditions ouvrières.

SCHUMACHER, Ernst F. (1973), Small is Beautiful: une société à la mesure de l'homme, Paris, Seuil.

StebBins, Robert A. (2000), The French Enigma: Survival and Development in Canada's Francophone Societies, Calgary, Detselig Enterprises.

THÉRIAULT, Joseph Yvon (1995), Lidentité à l'épreuve de la modernité: écrits politiques sur l'Acadie et les francophonies canadiennes minoritaires, Moncton, Éditions d'Acadie.

Thériault, Joseph Yvon (dir.) (1999), Francophonies minoritaires au Canada : l'état des lieux, Moncton, Éditions d'Acadie.

ThÉriault, Joseph Yvon (2003), " De l'école de la nation aux écoles communautaires ou de l'école d'en haut à l'école d'en bas ", dans Réal Allard (dir.), Actes du colloque pancanadien sur la recherche en éducation en milieu francophone minoritaire : bilan et prospectives, Moncton, Centre de recherche et développement en éducation; Québec, Association canadienne d'éducation de langue française, p. 47-55.

ThÉRIAUlt, Joseph Yvon (2004), "1604: la mémoire se joue de l'Acadie ", dans Michel Venne (dir.), L'Annuaire du Québec, Montréal, Fides, p. 673-677.

VAillanCOURT, Jean-Guy (2004), "Action 21 et le développement durable : après Rio 1992 et Johannesburg 2002 ", dans Louis Guay et al. (dir.), Les enjeux et les défis du développement durable: connaître, décider, agir, Québec, Presses de l'Université Laval, p. 37-57.

ZACCAİ, Edwin (2002), Le développement durable : dynamique et constitution d'un projet, Bruxelles, Presses interuniversitaires européennes. 